\title{
EVOLUTION OF NOVA 1934 DQ HERCULIS OVER 40 YEARS
}

\author{
E.S.DMITRIENKO \\ Crimean Astrophysical Observatory \\ dmitrien@crao.crimea.ua
}

We present the results of the analysis of the photometrical behaviour of Nova 1934 DQ Her for a time interval about 40 years based on our data and those published by different authors. Our observation were done in UBVRI in 1982-1997 during apptoximatly 170 nights. They give the possibility of investigating different types of the activity of the old nova with amplitudes of brightness variability from several tenths of magnitudes to $1^{m}$. The light oscillations on a time-scale of either several days or several dozens of days would be conditioned by the change of the velocity of the accretion from the disc on the white dwarf caused by the intrinsic disc instability or by the irregular receiving of matter from the secondary component of the system - a red dwarf. The cyclic change of the light of DQ Her on a time-scale of about several years could be the reaction of the accretion disc in response to the activity of the secondary star. 